\title{
MONOTONICITY OF FUNCTIONS INVOLVING Q-GAMMA FUNCTIONS
}

\author{
CHRYSi G. KOKOLOGIANNAKI
}

Abstract. We give monotonicity properties of some functions involving q-Gamma functions. The results are q-analogue of results concerning Gamma function and generalize some known results.

Mathematics subject classification (2010): 33B15, 33D05, 26D07, 24A48.

Keywords and phrases: q-Gamma function, monotonicity properties, inequalities.

\section{REFERENCES}

[1] M. Abramowitz, I. A. Stegun (Eds.), Handbook of Mathematical Functions with Formulas and Mathematical Tables, Applied Mathematics Series, Vol. 55, fourth ed., with corrections, National Bureau of Standards, Washington, 1965.

[2] H. AlZer, A. Z. GRinshPAn, Inequalities for the gamma and q-gamma functions, J. Approx. Theory, 144 (2007), 67-83.

[3] C. P. Chen, Inequalities for the Polygamma functions with Applications, General Mathematics, Vol. 13, 3 (2005), 65-72.

[4] C. P. CHEN, Complete monotonicity and logarithmically complete monotonicity properties for the gamma and psi functions, J. Math. Math. Anal. Appl., 336 (2007), 812-822.

[5] N. Elezovic, C. Giordano, J. Pecaric, A geometric mean inequality and some monotonicity results for the q-Gamma function, Mathematical Inequalities and Application, Vol. 1, 2 (1998), 253258.

[6] N. Elezovic, C. Giordano, J. PeCaric, Convexity and q-Gamma function, Rendiconti del Circolo Matematico di Palermo, Serie II, Tomo XLVIII (1999), 285-298.

[7] C. GIORdAno, A. LAFORGIA AND J. PeCARIC, Unified treatment of Gautshi-Kershaw type inequalities for the gamma function, J. Comp. Appl. Math., 99 (1998), 167-175.

[8] M. E. H. Ismail, L. LorCh AND M. E. Muldoon, Completely monotonic functions associated with the Gamma function and its q-analogues, J. Math. Math. Anal. Appl., 116 (1986), 1-9.

[9] S. Koumandos, Monotonicity of some functions involving the gamma and psi functions, Mathematics of Computation, 77, 264 (2008), 2261-2275.

[10] T. MANSOUR, Some inequalities for the q-Gamma function, Journal of inequalities in pure and Applied Mathematics, 9, 1 (2008), Article 18, 4pp.

[11] J. Pecaric, G. Allasia And C. Giordano, Convexity and the Gamma function, Indian Journal of Mathematics, B.N. Prasad birth centenary commemoration Volume, 41, 1 (1999) 79-93.

[12] A. SH. SHABANI, Generalization of some inequalities for the q-Gamma function, Annales Mathematicae et Informaticae, 35 (2008), 129-134. 